\title{
Regional cerebral blood flow and Cingulate island sign in dementia with Lewy bodies
}

Hideki Matsuoka M.D. 1), Hitoshi lida 1), Koji Ogomori 1), Hiroaki Kawasaki 1), Koichi Takano 2), Shigeki Nagamachi ${ }^{2)}$

1. Department of Psychiatry, Faculty of Medicine, Fukuoka University, Japan

2. Department of Radiology, Faculty of Medicine, Fukuoka University, Japan

E-mail:

peaceful.smile.face@gmail.com

\section{Conclusion}

\section{Cingulate island sign score (CIScore) is useful regarding to differentiate between Alzheimer's disease (AD) and dementia with Lewy bodies (DLB).}

\section{Introduction}

It is clinically important to distinguish DLB from AD. The relative preservation of cingulate glucose metabolism in the posterior cingulate gyri versus that in the precuni, known as the cingulate island sign (CIS), in patients with DLB compared with AD is supposed to be highly specific for diagnosing DLB. CIS has been detected using [18F]fluorodeoxyglucose positron emission tomography ([18F]FDG-PET) but not using brain perfusion single-photon emission computed tomography (SPECT). Actually, brain perfusion SPECT is not only more commonly used but is also a more economical modality compared with [18F]FDG PET. However, CIS on SPECT is difficult to distinguish DLB from AD at first glance.

\section{CIS on FDG-PET}

The white arrow points to posterior cingulate cortex on FDG PET, demonstrating cingulate island sign of DLB.

\section{CIS on SPECT}

The red arrow points to posterior cingulate cortex on SPECT, and blue arrow points to hypoperfusion in occipital lobe of DLB. On the other hand, AD cases have hypoperfusion in posterior cingulate cortex and preservation of occipital lobe.
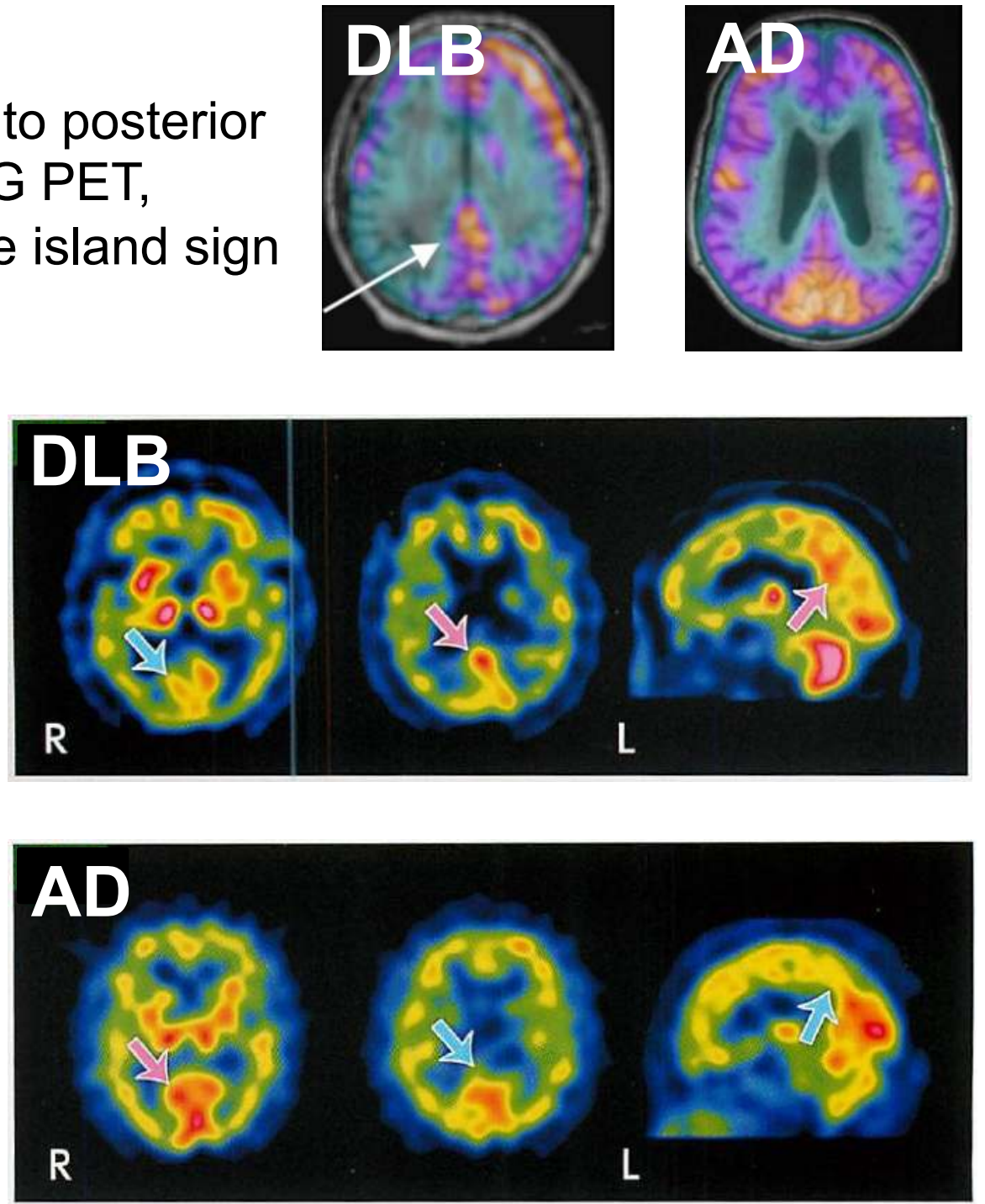

\section{Recent findings}

Recently, "CIScore (Cingulate Island Sign Score) ", whose score is defined by using the Easy z-score imaging system (eZIS) analysis (Fujifilm RI Pharma Co., Ltd., Tokyo, Japan) software, is proposed to be very effective index for differential diagnosis of dementia. This software (eZIS) analyzes the patient's images by voxel-to-voxel comparisons with those from age-matched normal database, and then generated Z-score images as a more objective and reliable assessments of functional abnormalities.

CIScore $=\frac{\begin{array}{c}\text { Total positive Z score values within mainly } \\ \text { occipital lobe (DLB specific area) }\end{array}}{\begin{array}{c}\text { Total positive Z score values within mainly } \\ \text { posterior cingulate cortex (AD specific area) }\end{array}}$

It is reported that the accuracy, sensitivity, and specificity of these new CIS ratios were $84.6,92.3$, and $76.9 \%$, respectively, with a threshold value less than 0.281 for CIScore, and the area under the receiver operating characteristic (ROC) curve (AUC) was 0.882

\section{Goal}

This study was conducted to examine the differences between $A D$ and DLB by investigating regional cerebral blood flow (rCBF) and CIS by using brain perfusion SPECT.

\section{Methods}

We recruited subjects who included a diagnosis of probable DLB $(n=28)$, possible DLB $(n=20), A D(n=37)$, and normal control. All subjects underwent SPECT scans using technetium-99m-ethyl cysteinate dimer (ECD). We examined the effectiveness of this CIScore for differential diagnosis between AD and DLB. We evaluated rCBF and CIS. Data were analyzed using Statistical Parametric Mapping software.

\begin{tabular}{|c|c|c|c|c|c|}
\hline \multicolumn{6}{|c|}{ Results } \\
\hline & $\begin{array}{l}\text { CIS } \\
\text { positive } \\
\text { rate } \\
\text { CIScore } \\
<0.281\end{array}$ & $\begin{array}{c}\text { mean } \\
\text { CIScore }\end{array}$ & & $\begin{array}{l}\text { Z-score } \\
\text { posterior } \\
\text { gulate cortex }\end{array}$ & $\begin{array}{c}\text { Z-score } \\
\text { occipital lobe }\end{array}$ \\
\hline AD & $\begin{array}{c}\mathbf{4 8 . 6 \%} \\
18 / 37\end{array}$ & $\left.\begin{array}{c}0.40 \\
\pm 0.42\end{array}\right]$ & & $\begin{array}{l}1871.350 \\
{[873.314}\end{array}$ & $\begin{array}{c}6518.341 \\
\pm 3413.228\end{array}$ \\
\hline $\begin{array}{c}\text { Probable } \\
\text { DLB }\end{array}$ & $\begin{array}{c}92.9 \% * \\
26 / 28\end{array}$ & $\begin{array}{l}0.15 \\
\pm 0.08\end{array}$ & $*$ & $\begin{array}{l}1366.545 \\
\pm 941.791\end{array}$ & $\begin{array}{r}9507.691 \\
\pm 5808.952\end{array}$ \\
\hline $\begin{array}{c}\text { Possible } \\
\text { DLB }\end{array}$ & $\begin{array}{c}\mathbf{8 5 . 0} \% \\
17 / 20\end{array}$ & $\begin{array}{l}0.17 \\
\pm 0.09\end{array}$ & & $\begin{array}{r}1329.937 \\
\pm 683.903\end{array}$ & $\begin{array}{c}8177.811 \\
\pm 3005.265\end{array}$ \\
\hline $\begin{array}{c}\text { AD } \\
\text { (CIS positive) }\end{array}$ & $\begin{array}{l}100 \% \\
18 / 18\end{array}$ & $\begin{array}{l}0.19 \\
\pm 0.05\end{array}$ & & $\begin{array}{l}1385.764 \\
\pm 524.497\end{array}$ & $\begin{array}{c}7801.579 \\
\pm 3240.556\end{array}$ \\
\hline
\end{tabular}

The accuracy, sensitivity, and specificity of these CIS ratios were $72.9,89.6$, and $51.4 \%$, respectively, with a threshold value less than 0.281 for CIScore.

\section{Discussion}

In data aggregated at our facility, CIS was often positive in DLB compared with $A D$. However, when comparing the group that became positive CIS among the AD group and the DLB group, it was indistinguishable from DLB by CIS finding only. It is important not to conduct differential diagnosis only with CIS, but to comprehensively diagnose refer to other brain imaging. In addition, we defined that the threshold value of CIScore was 0.281 , but the threshold value may not been the best one. It is necessary to make effort to increase the number of case series in a more proactive manner, such as keeping track of the number of dementia cases and increases in the number of cases at each hospital and securing the number of cases in collaboration with multiple institutions.

\section{Reference}

Imabayashi $\mathrm{E}$ et al. Validation of the cingulate island sign with optimized ratios for discriminating dementia with Lewy bodies from Alzheimer's disease using brain perfusion SPECT. Annals of Nuclear Medicine. August 2017, Volume 31, Issue 7, pp 536-543. 